\title{
A semi-analytical model for droplet dynamics on the GDL surface of a PEFC electrode
}

\author{
Alex Jarauta ${ }^{\mathrm{a}, *}$, Marc Secanell ${ }^{\mathrm{b}}$, Jordi Pons-Prats ${ }^{\mathrm{a}}$, Pavel Ryzhakov ${ }^{\mathrm{a}}$, Sergio \\ R. Idelsohn ${ }^{\mathrm{a}, \mathrm{c}}$, Eugenio Oñate ${ }^{\mathrm{a}}$ \\ ${ }^{a}$ Centre Internacional de Mètodes Numèrics en Enginyeria (CIMNE), Gran Capitán s/n, \\ 08034 Barcelona, Spain \\ ${ }^{b}$ Energy Systems Design Lab (ESDLab), University of Alberta, Edmonton, Canada \\ ${ }^{c}$ Institució Catalana de Recerca i Estudis Avançats (ICREA), Barcelona, Spain
}

\begin{abstract}
Water management is one of the key factors in Proton Exchange Fuel Cell (PEFC) performance. The water produced within the fuel cell is evacuated through the gas channels, but at high current densities water can block the channel, thus limiting the current density generated in the fuel cell. A semianalytical model of a water droplet emerging from a gas diffusion layer pore in a PEFC channel is developed. The transient model contains a detailed adhesion and drag force estimation model. Results show that the predicted values for both drag and surface tension force are higher than the results found in literature. The results for the detachment force are consistent with the experimental data available. Higher air velocity values lead to more deformation of the droplet and oscillation with lower frequency but higher amplitude. Similar effects have been identified when the liquid mass flow is increased, leading to faster detachment of the droplet.
\end{abstract}

Keywords: Fuel cells, Droplet dynamics, Adhesion force, Drag force

\section{Introduction}

Water management is a key limiting factor of PEFCs performance [1]. Water and heat are produced in the oxygen reduction reaction (ORR) in the cathode catalyst layer (CL) of the PEM fuel cell. A fraction of this water is used to humidify the membrane and the exceeding water has to be evacuated through the pores of the gas diffusion layer (GDL). When it emerges from the pores into the gas channels it may form droplets, films or slug flow depending on the working conditions [2]. The present study improves previous models used to understand the formation of droplets on the GDL surface [3], [4], [5], [1], [6], [7].

*Corresponding author. Tel.: +34 934134188

Email: ajarauta@cimne.upc.edu

Preprint submitted to International Journal of Hydrogen Energy

December 1, 2014 
Chen et al. [3] published an analytical model of a water droplet in a PEFC cathode channel based on a macroscopic force balance. This study was the first to study a water droplet subjected to an airflow and laying on a porous surface [3]. The analytical study was steady-state since it was based on the equilibrium of the forces acting on the droplet. The main drawback of the model was that it did not take into account the effect of pinning and that the study was steadystate.

Kumbur et al. [8] developed a model of a water droplet in the flow channel of a PEFC in order to predict its detachment. By means of an experiment, they found a model that related the content of Teflon in the GDL surface and the surface tension. They improved Chen's work but they still could not characterize the droplet geometry, the drag coefficient or the area coverage of the GDL surface. This study was also steady-state, so no transient analytical model was yet developed.

An exhaustive analysis of the static and dynamic shape of the water droplet was done by Esposito et al. [9]. This study improved upon the previous models by providing a consistent analytical model that accounted for the effects of the forces on the droplet as a function of time. Their predictions did not agree well

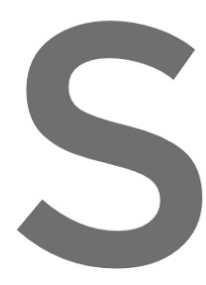

with their
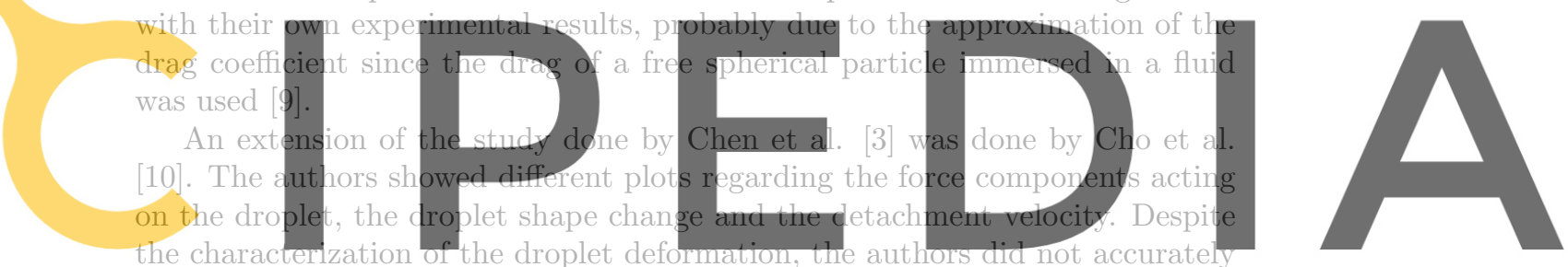

identify the deformed shape of the droplet and the evolution of the contact line

Register for free at httep\$//WWw wuseipedia.com to download the version without the watermark

In addition to the analytical models above, researchers have also studied

droplet dynamics using numerical methods. A two-dimensional numerical simulation was developed by Zhu et al. [7] using the Volume of Fluid (VOF) method [11]. Theodorakakos et al. [4] developed a numerical simulation of a droplet deformed by an airflow laying on the GDL surface. The results gave accurate plots of the deformed shape of the droplet at different time steps. Zhu et al. [1] also developed a three-dimensional numerical simulation. It was an extension of their previous work and it was closer to the actual case since more parameters were taken into account. Results showed that water removal was achieved with a hydrophobic GDL surface. VOF models, even though they are very accurate, are time consuming and difficult to integrate with full Membrane Electrode Assembly (MEA) models.

This article presents a semi-analytical model of droplet dynamics on the GDL surface of a PEFC. An analytical approach is used because it can be easily integrated with an MEA model. The main contribution of this work is to develop an analytical model that includes the following improved physical descriptions:

a) the adhesion force is obtained using the formula proposed by Extrand et 


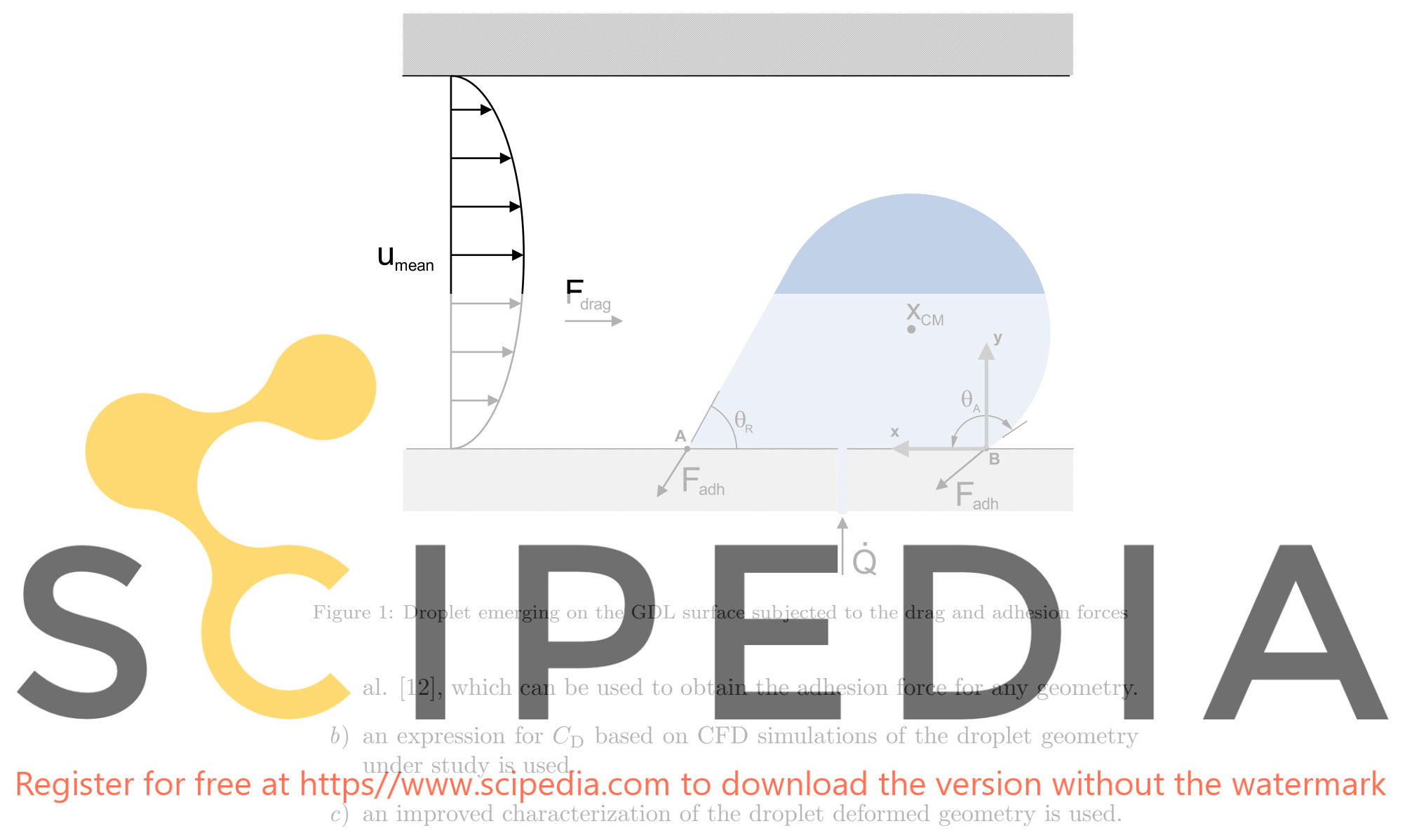

\section{Governing equations}

The following model assumes that gravity and viscous effects are negligible compared to the surface tension force. Figure 1 shows a schematic view of the studied problem. A water droplet emerges from a pore into a channel with a constant volume flow $\dot{Q}$. The air flows with a fully developed velocity profile and an average velocity of $u_{\text {mean }}$. As the air flows around the droplet, it exerts a drag force, $F_{\text {drag }}$, on the droplet surface, which tends to deform it. Since this force breaks the droplet equilibrium, the adhesion force acts on the opposite direction of the air drag, thus taking the droplet to a new equilibrium state. This unbalance of the droplet leads to droplet oscillations.

This problem should be modelled with a transient model since the equilibrium state depends on the previous state of the droplet. The equation that characterizes the balance of forces acting on the droplet is Newton's second law 
applied to the $\mathrm{x}$-coordinate of the droplet center of mass,

$$
m \frac{d^{2} x_{\mathrm{CM}}}{d t^{2}}=F_{\mathrm{adh}}-F_{\mathrm{drag}}
$$

where $m$ is the mass of the droplet and is a function of time, $x_{\mathrm{CM}}$ is the $\mathrm{x}$ coordinate of the center of mass and $F_{\text {adh }}$ and $F_{\text {drag }}$ are the adhesion and drag forces, respectively. These forces are described by the following equations,

$$
F_{\text {drag }}=\frac{1}{2} \rho_{\text {air }} u_{\text {air }}^{2} C_{\mathrm{D}} A
$$

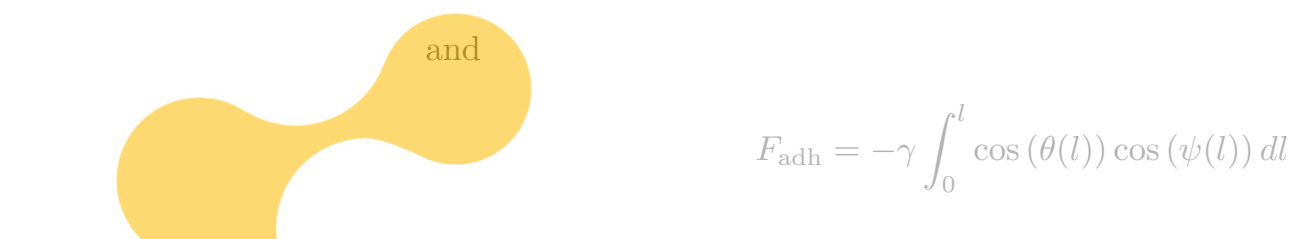

where $\rho_{\text {air }}$ is the air density, $C_{\mathrm{D}}$ is the drag coefficient, $A$ is the frontal projected area of the droplet and $\gamma$ is the surface tension between the air and the water.
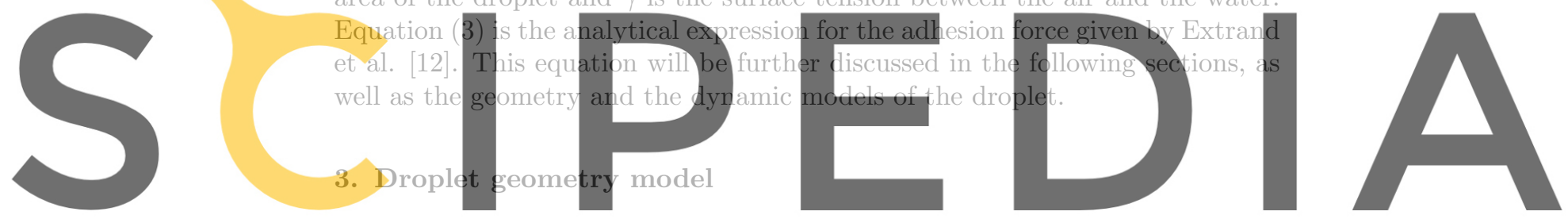

The droplet geometry and forces acting on the droplet are fully coupled, so

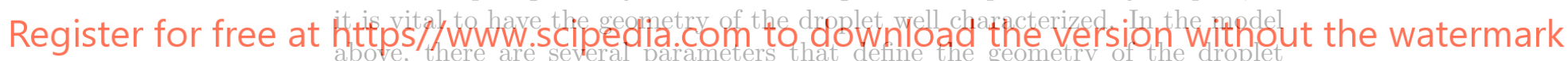
such as the advancing and receding angles, the frontal area and the droplet thickness. In order to develop analytical expressions for the geometry of the droplet, the droplet is idealized as a semi-spherical cap together with a cone shape to model pinning [9], and these parameters can be obtained as a function of the droplet volume. Even though the idealized geometry cannot represent the complex geometries that might form, e.g. slugs, it contains mechanisms to account for pinning and contact angle hysteresis. This idealized geometry model is based on the one developed by Esposito et al. [9] with several corrections and improvements.

The geometry model uses the water volume flow $\dot{Q}$ and an initial chord length $c$ (distance $\mathrm{AB}$ in Figure 1) as input parameters to obtain the rest of the variables at every time step. Considering a constant chord and a constant area of the middle section for every deformation state, it is possible to find an expression of the chord length $c$ of the droplet as a function of the contact angles:

$$
c=\frac{R}{\sin \theta_{\mathrm{R}}}\left(1+\sin \theta_{\mathrm{A}} \sin \theta_{\mathrm{R}}-\cos \theta_{\mathrm{A}} \cos \theta_{\mathrm{R}}\right)
$$


and also an expression for the middle section area of the droplet,

$$
A=\frac{R^{2}}{2 \sin \theta_{\mathrm{R}}}\left(\left[\theta_{\mathrm{A}}+\theta_{\mathrm{R}}-\sin 2 \theta_{\mathrm{A}}+\sin \Delta\right] \sin \theta_{\mathrm{R}}+4 \sin ^{3}\left(\frac{\Delta}{2}\right) \sin \left(\frac{\theta_{\mathrm{A}}+\theta_{\mathrm{R}}}{2}\right)\right)
$$

where $\Delta=\theta_{\mathrm{A}}-\theta_{\mathrm{R}}$ is the contact angle hysteresis. The equations of the droplet height and perimeter are shown below:
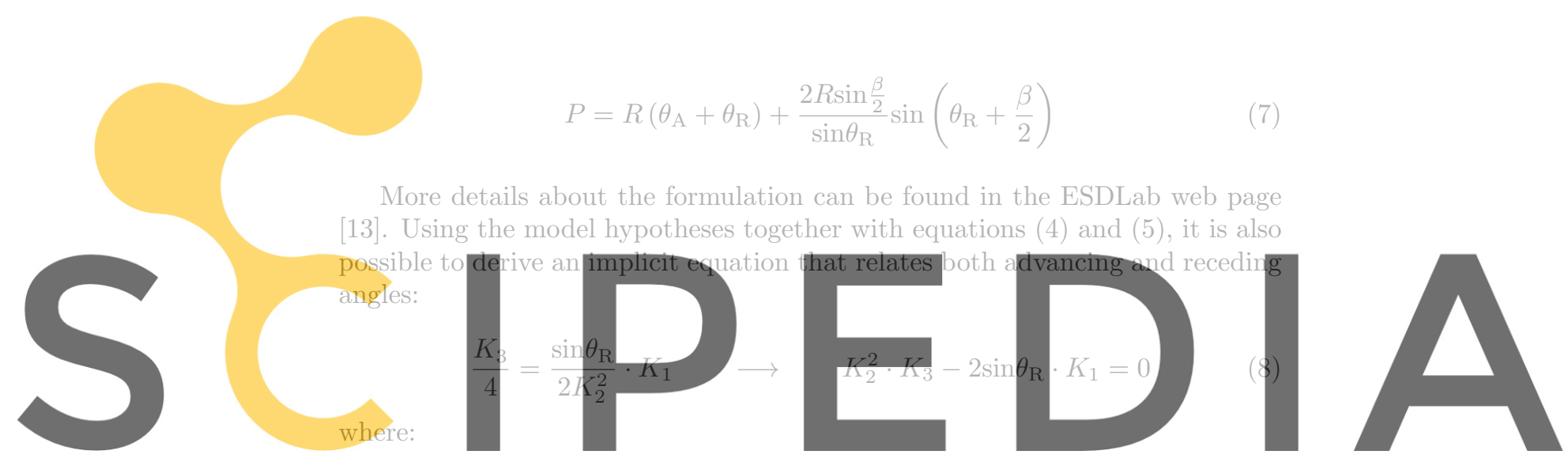

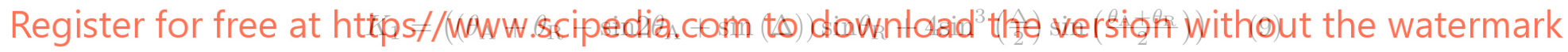

$$
\begin{gathered}
K_{2}=1+\sin \theta_{\mathrm{A}} \sin \theta_{\mathrm{R}}-\cos \theta_{\mathrm{A}} \cos \theta_{\mathrm{R}} \\
K_{3}=\frac{1}{\sin ^{2} \theta_{\mathrm{S}}}\left(\theta_{\mathrm{S}}-\frac{\sin 2 \theta_{\mathrm{S}}}{2}\right)
\end{gathered}
$$

Equation (8) does not depend on the droplet size. Since it is impossible to find an explicit equation for the receding angle, a fitting function needs to be found in order to have an expression for this magnitude. A single expression to fit all the values of the receding angle would introduce a high estimation error for values of the advancing angle close to $\theta_{\mathrm{S}}$ because from $\theta_{\mathrm{A}}=2.39$ to 2.43 rad (values close to the static angle), the receding angle drops quickly, whereas from 2.43 to $\pi, \theta_{\mathrm{R}}$ decreases almost linearly.

Using equation (8), a set of 200 values has been generated to estimate a fitting curve in each interval, giving $\theta_{\mathrm{A}}$ values from $2.39 \mathrm{rad}$ to $\pi$ with an increment of $0.005 \mathrm{rad}$. Therefore, two different equations are proposed to estimate the receding angle based on the advancing angle. Both equations have been found using the commercial software CurveExpert Basic, which uses the 
Table 1: Constants used in the fitting curve of the advancing and receding angles relationship

\begin{tabular}{lcccc}
\hline$\theta_{\mathrm{A}}$ & $\mathrm{a}$ & $\mathrm{b}$ & $\mathrm{c}$ & $\mathrm{d}$ \\
\hline \hline$[2.39,2.43)$ & -0.5771 & 0.2414 & -0.9432 & 0.2195 \\
{$[2.43, \pi]$} & -1.5154 & 0.6500 & -1.1679 & 0.3164 \\
\hline
\end{tabular}

Levenberg-Marquardt algorithm to perform nonlinear regression. Using this fitting curve software, the best curve for both cases is:

$$
\theta_{\mathrm{R}}=\frac{a+b \theta_{\mathrm{A}}}{1+c \theta_{\mathrm{A}}+d \theta_{\mathrm{A}}^{2}}
$$

where the constants $a, b, c$ and $d$ have the values shown in Table 1.

In both cases, the Coefficient of Determination $R^{2}$ is higher than 0.999, which means that the proposed functions have an excellent fit with the numerical data.

\section{Droplet dynamics model}
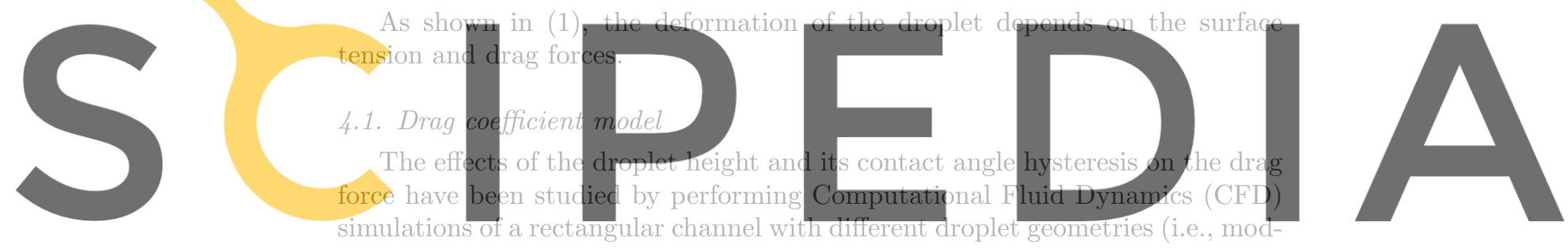

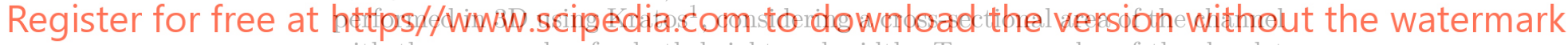
with the same value for both height and width. Two examples of the droplet geometries used in the numerical simulations are shown in Fig. 2.

In the simulations, the computational domain is a channel with a squared cross-sectional area of $1 \mathrm{~mm}$ per side and $50 \mathrm{~mm}$ long. The simulations have been done considering that the air is flowing in steady-state with a fully developed laminar profile for the velocity and an average value of $u_{\text {air }}$ (Table 2 in Section 4.3). The governing equations for the air's velocity and pressure are the stabilized continuum and momentum equations [15]. The boundary conditions are the following:

- Dirichlet boundary condition for the velocity at the inlet; fully developed laminar profile with average velocity $u_{\text {air }}$.

- Neumann boundary condition for the pressure at the outlet, i.e. no viscous stress (open boundary).

- No slip boundary condition to the rest of the walls and the droplet.

\footnotetext{
${ }^{1}$ Kratos Multi-Physics System is a C++ object oriented FE framework [14]
} 

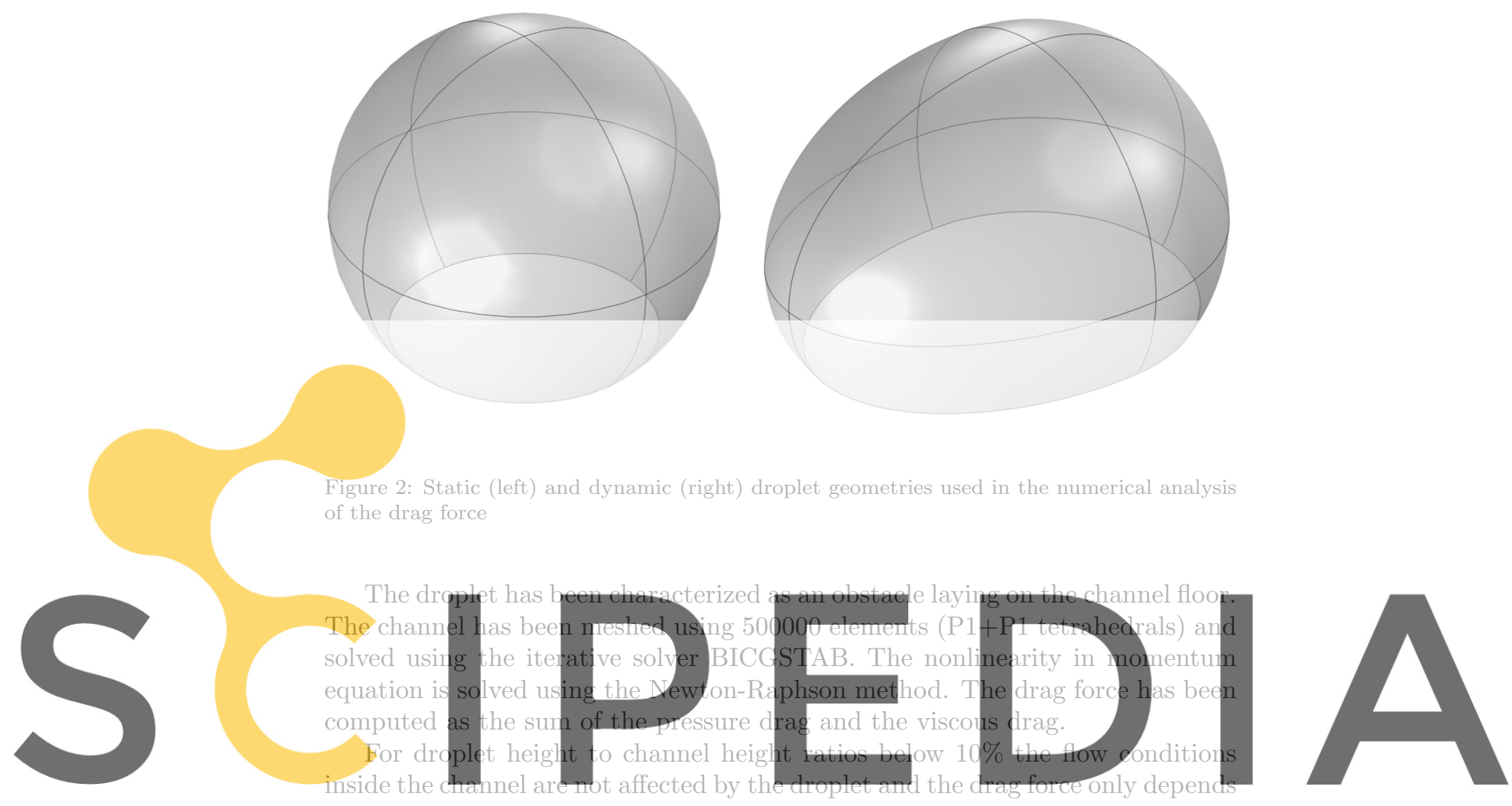

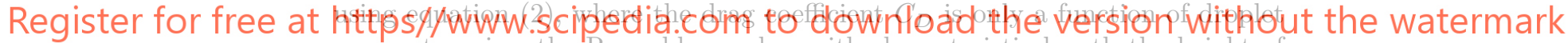
parameters, i.e. the Reynolds number with characteristic length the height of the droplet, $h$, and hysteresis contact angle, $\Delta$. Using CFD simulations, the drag coefficient is found to be

$$
C_{\mathrm{D}}=18.4-0.777 R e_{\mathrm{h}}-0.772 \Delta
$$

To obtain the coefficients for the equation above, simulations where performed and statistical regression showed that the three coefficients included in the model have p-values lower than 0.001 , and thus they are statistically significant in the model. Simulations with different channel heights and different fluid properties were performed and the equation is shown to hold.

For droplet height to channel height ratios above $10 \%$, the flow conditions in the channel are affected by the droplet. In this case, both droplet height and height of the channel influence the results. Furthermore, since the drag depends not only on the drop, equation (2) cannot be used. Several simulations were performed in this case and it was found that the drag force (expressed in $\mu \mathrm{N}$ ) 
could be approximated by:

$$
\begin{gathered}
F_{\mathrm{drag}}=5.81-4.82 R e_{\mathrm{H}-\mathrm{h}}+1.67 R e_{\mathrm{H}-\mathrm{h}}^{2}-0.29 R e_{\mathrm{H}-\mathrm{h}}^{3}+ \\
+0.03 R e_{\mathrm{H}-\mathrm{h}}^{4}-8.66 \times 10^{-4} \cdot R e_{\mathrm{H}-\mathrm{h}}^{5}-1.43 \Delta \mathrm{e}^{-0.959 R e_{\mathrm{H}-\mathrm{h}}}
\end{gathered}
$$

The exponential term in equation (14) means that the difference between the static and deformed cases increases as $H-h$ decreases. The coefficients from equation (14) were obtained by running simulations with air fluid properties, droplet heights from 0.01 to $8 \mathrm{~mm}$ and channel geometries from 0.1 to $10 \mathrm{~mm}$.

In order to estimate the generality of the equation, different fluid properties were also studied. Results showed that the drag force obtained from the CFD simulation and the one predicted from the equation above differed by less than $1 \%$.

\subsection{Adhesion force model}

The surface tension force acts on the droplet when the droplet is taken away

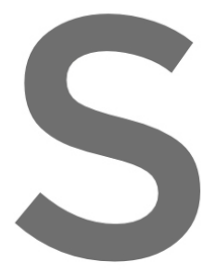
from its resting position. The force is proportional to the droplet deformation

\section{Celestini and Kofm:} as [16]:
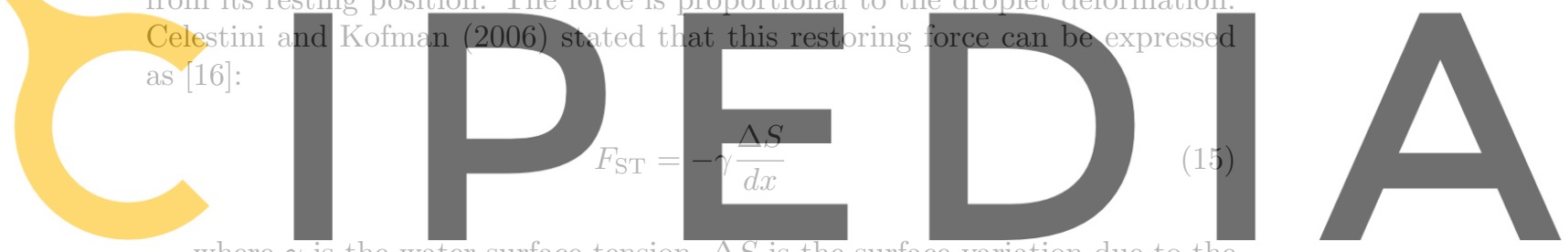

deformation and $d x$ is the displacement of the center of mass from its original

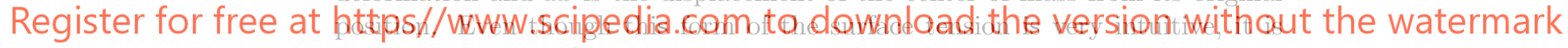
an approximation of the actual phenomena. Moreover, the spring model can be misunderstood since the spring force acts on the center of mass, whereas the adhesion force is applied at the contact line with the surface. Thus, in this work the adhesion force of the droplet is computed numerically using equation (3) [12], where $\theta$ is the contact angle and $\psi$ is the angle between the surface tension force in the $x y$ plane and the $x$ axis, as shown in Figure 3.

This expression is integrated numerically to obtain the approximated value of the adhesion force at every deformation state [17]. As Antonini et al. showed in their work [17], for hydrophobic surfaces with static contact angle greater than $90^{\circ}$ the contact line of a deformed droplet can be described as a double ellipse. The proposed model considers that the $y$ semi-radius remains constant for the two ellipses, and the sum of the two $x$ semi-radii is equal to the droplet chord. Using the geometry and numerical integration, equation (3) is solved at every time step. In our model, the contact angle distribution in every semiellipse has been considered linear with respect to the $x$ direction, going from the value of the static contact angle to the value of the advancing or receding angle in each case. 


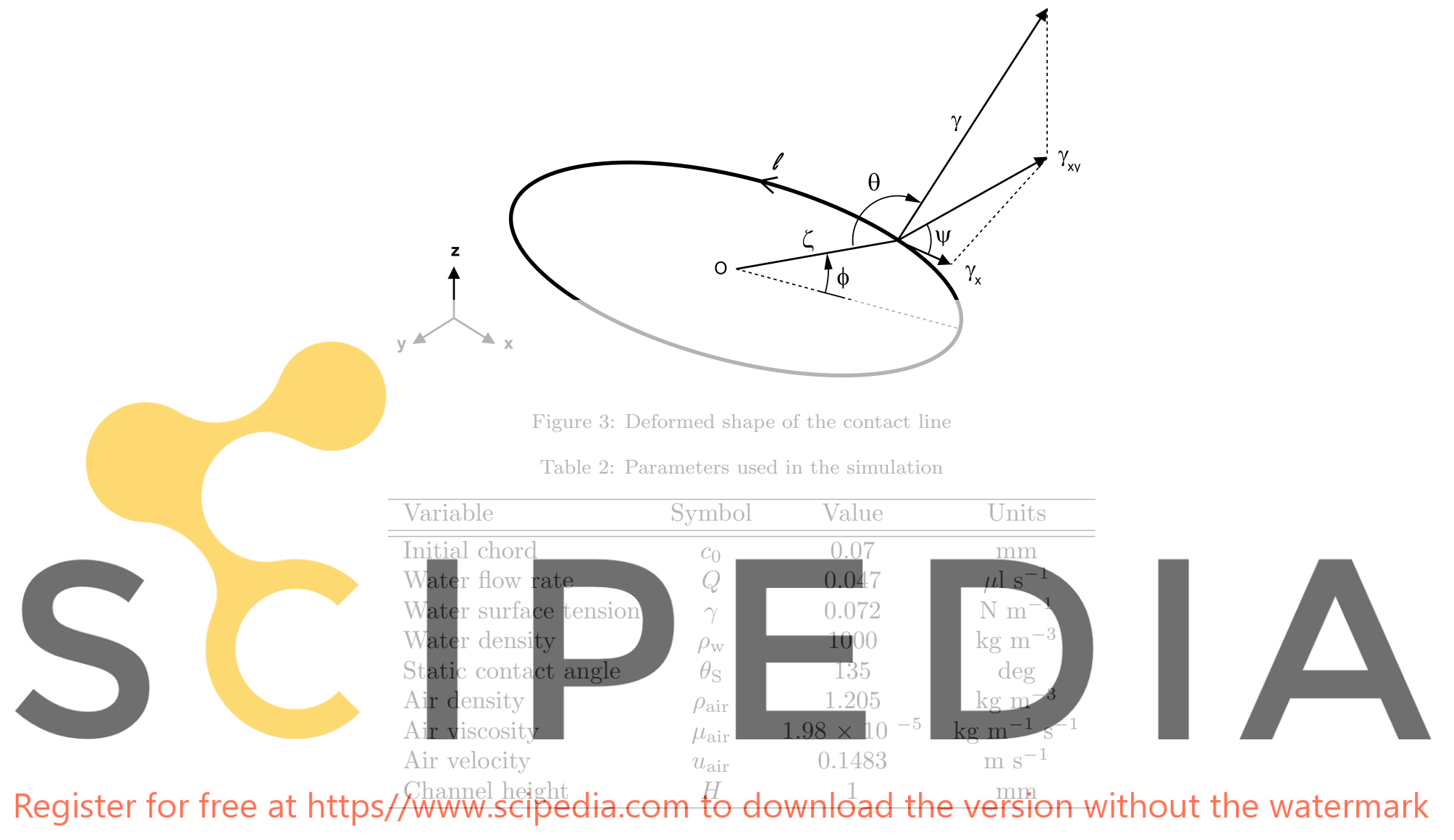

\subsection{Boundary conditions}

In order to solve the differential equation (1), initial conditions must be imposed for the x-coordinate of the center of mass. Since equation (1) is a second order differential equation, the following two initial conditions are imposed,

$$
\begin{gathered}
\left.x_{\mathrm{CM}}\right|_{t=0}=\frac{c_{0}}{2} \\
\left.\frac{d x_{\mathrm{CM}}}{d t}\right|_{t=0}=\left.v_{\mathrm{CM}}\right|_{t=0}=0
\end{gathered}
$$

In other words, at $t=0$ the droplet is static. The term $c_{0}$ refers to the droplet chord at the beginning of the simulation. The rest of the parameters used in the simulation are described in Table 2.

The velocity of the air and the water injection rate have been obtained considering that the fuel cell channel has a $1 \mathrm{~mm} \times 1 \mathrm{~mm}$ cross-sectional area and $5 \mathrm{~cm}$ length, the current density is $1 \mathrm{~A} \mathrm{~cm}^{-2}$ and a stoichiometric rate of one. Equation (1) and the aforementioned boundary conditions are implemented in MATLAB and solved using an in-house 4th order Runge-Kutta method. 


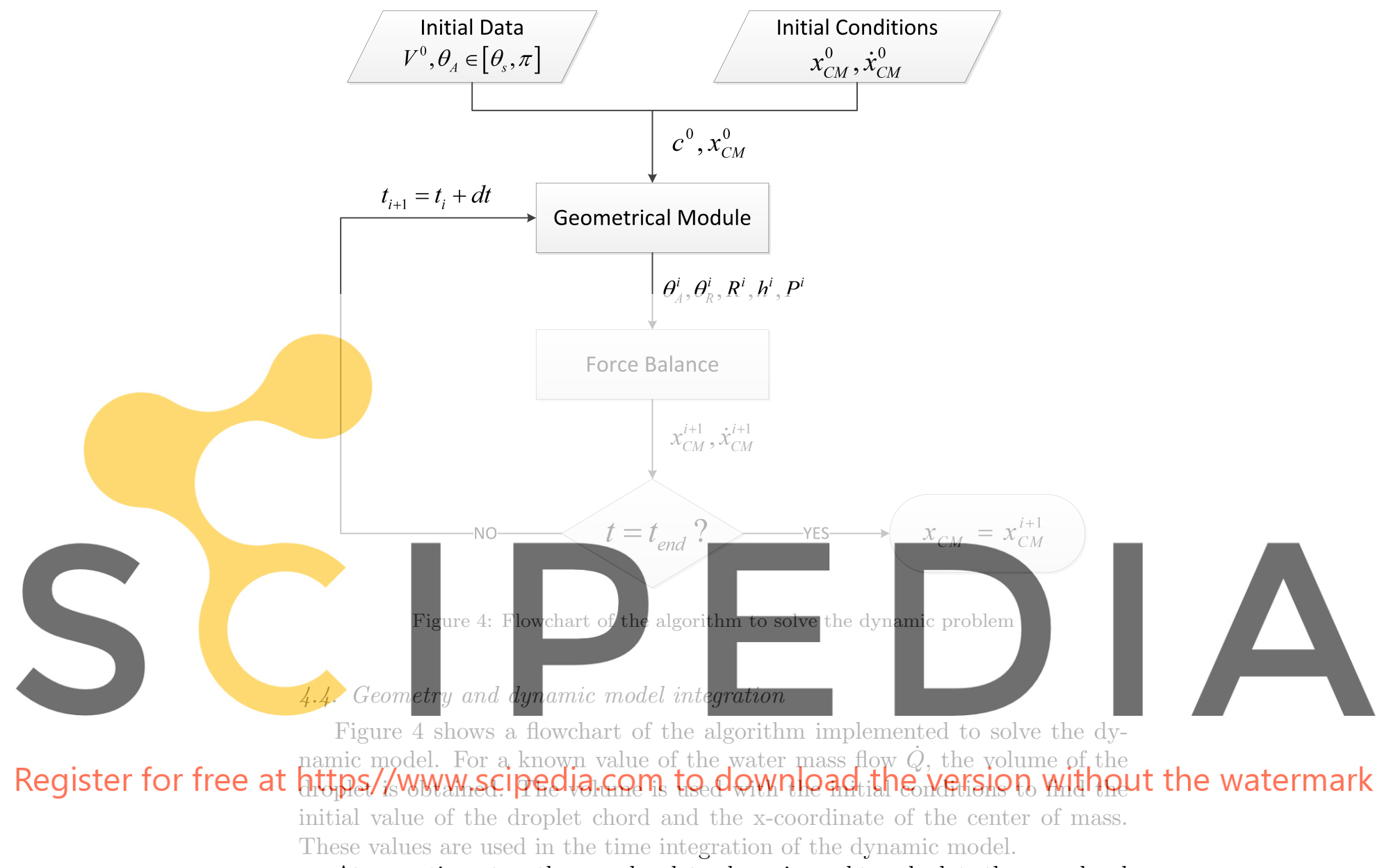

At every time step, the new droplet volume is used to calculate the new chord length and the $\mathrm{x}$-coordinate of the center of mass. These parameters are used in the geometrical model to find the contact angles and the rest of geometrical variables. The forces at the current time step are computed using the known geometry of the droplet. Then, the force balance results in the $\mathrm{x}$-coordinate of the center of mass and its velocity at the next time step. This process is repeated until the final time step is reached.

\section{Results and discussion}

In this section, the most important results regarding the droplet dynamics are displayed. The total time of the simulation is $1 \mathrm{~s}$, using a droplet that grows constantly. The velocity of the air flowing around the droplet is $0.1483 \mathrm{~m} \mathrm{~s}^{-1}$ (Table 2). The time step size chosen for this simulation is $10^{-4} \mathrm{~s}$, and the total computational time is about $2 \mathrm{~min}$. 


\subsection{Validation}

The semi-analytical model is validated by reproducing several experiments. Milne et al. [18] measured the critical drag force needed to detach a sessile droplet from a hydrophobic surface. Different droplet volumes were considered in order to obtain a relationship between volume and critical drag force. The experiment is reproduced here by first computing the droplet geometry for every volume, setting a value of 0 for the water injection rate $(\dot{Q}=0)$ and increasing the velocity of the air until the detachment condition is fulfilled. According to Milne's work, the droplet detaches when the contact angle hysteresis is greater than 30 degrees [18] (results obtained for a GDL surface with Teflon). The drag force when the droplet detaches is easily obtained from either equation (2) or (14). Figure 5 (a) shows the comparison between the experimental results from Milne, the predicted values with the proposed model, and the values obtained with the spring model in reference [9].

The values predicted with the current semi-analytical model are in good agreement with the experimental data. The values obtained with a spring model largely underpredict the critical drag force. The difference between the experimental data and the predictions from the proposed model is larger at higher droplet volumes. As the droplet grows, gravity and viscous effects become more important. In the present model gravity effects are neglected, which can dominate for large droplet sizes. However, the proposed model predicts well the drag force necessary for droplet detachment for droplet volumes up to $100 \mu \mathrm{L}$.

In order to further validate the proposed model predictions, predicted droplet size before detachment is compared to the experimental results reported by Zhang et al. [19]. Zhang et al. [19] measured the droplet size before detachment for droplets at different air velocity. In the simulations, the air velocity is fixed and the droplet is allowed to grow until detachment takes place. Detachment occurs when the transient hysteresis angle reaches 30 degrees. Figure 5 (b) compares the experimental data in reference [19] and the predicted droplet size according to the proposed model, using Milne's conditions for the critical hysteresis angle. Results obtained are in good agreement with the experimental data in reference [18] Both results have the same tendency: the higher the air velocity, the lower the critical droplet diameter. Thus, the critical hysteresis angle can be taken as $30^{\circ}$.

\subsection{Evolution of the center of mass, hysteresis angle and forces}

Figure 6(a) shows the effect of air velocity on the droplet center of mass for a droplet in a $1 \mathrm{~mm} \times 1 \mathrm{~mm}$ rectangular channel typical of a fuel cell. For

large flow rates, the center of mass position is well below the center of mass of the static droplet indicating that the center of mass approaches the advancing contact point (see point $B$ in Figure 1). When the velocity of the air is 0 , the xcoordinate of the droplet center of mass evolves without almost any oscillation, as expected. It has been observed, e.g. references [9] [4], that the higher the air velocity, the bigger the amplitude of the droplet oscillations, i.e. the droplet is more unstable and is prone to detach from the GDL surface. The frequency of the oscillations however remains almost constant. 


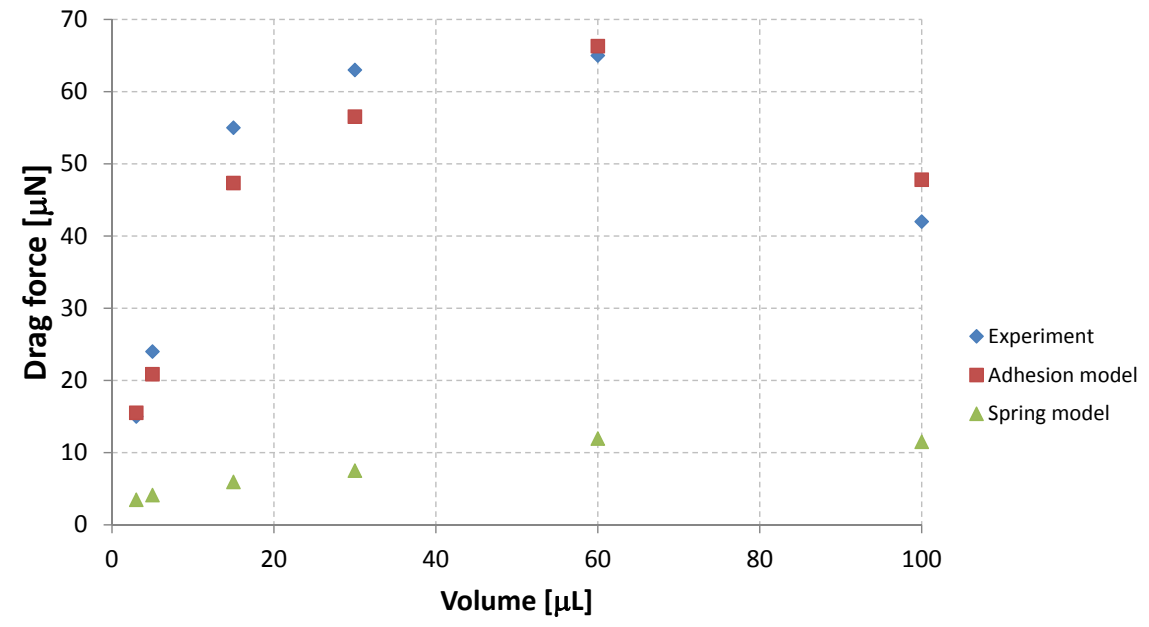

(a) Critical drag force for detachment vs droplet volume

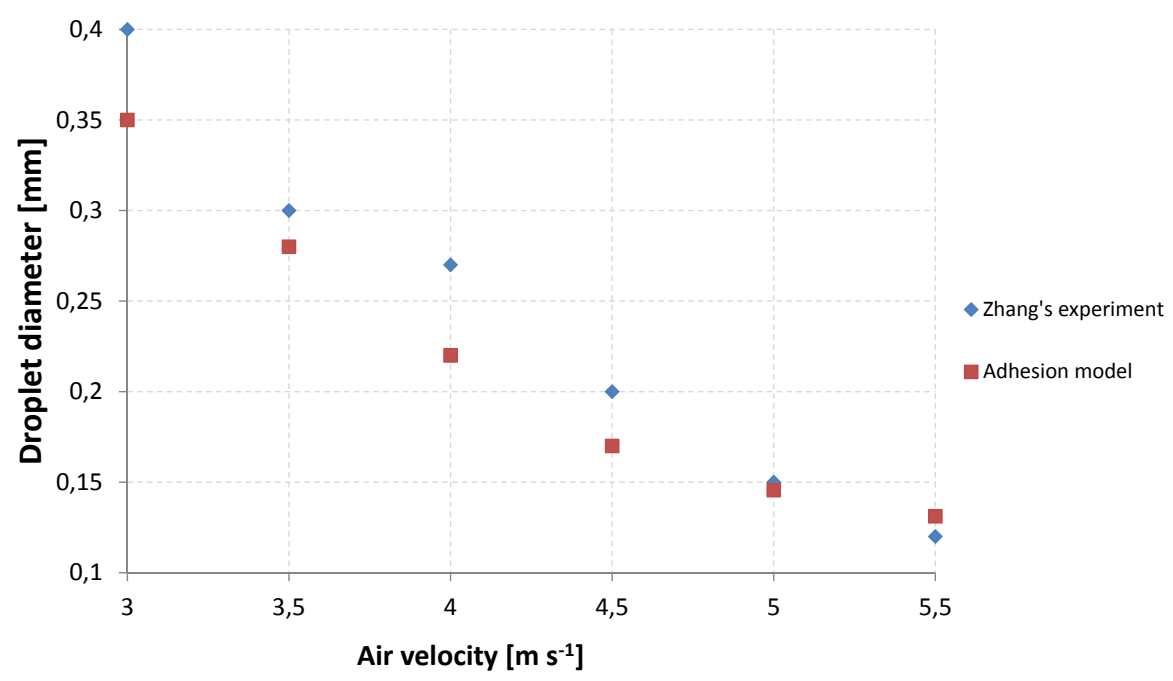

(b) Critical droplet diameter vs air velocity

Figure 5: Validation results of the semi-analytical model 


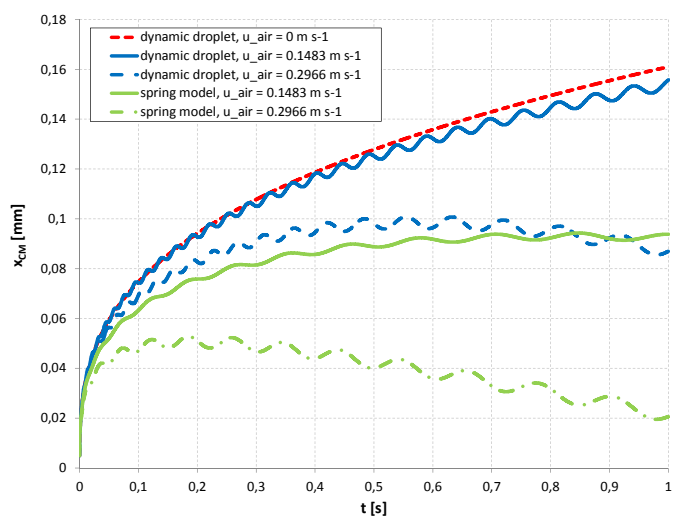

(a) Evolution of center of mass $\mathrm{x}$-coordinate
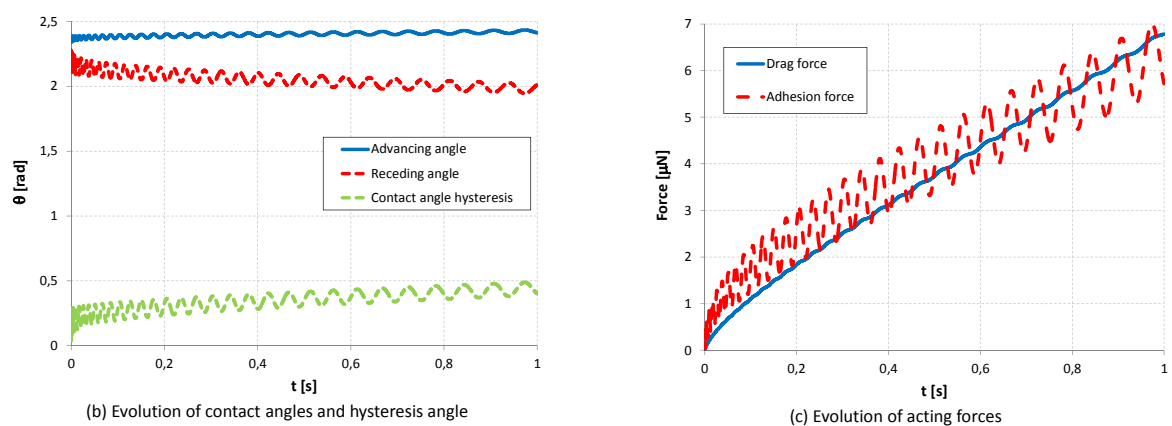

Figure 6: Evolution of the center of mass x-coordinate, contact angles and forces acting on the droplet 
The green lines correspond to the spring model for the adhesion force proposed in reference [9]. The proposed model predicts higher adhesion forces, resulting in a stiffer droplet with less deformation. Therefore, the droplet is less prone to detach from the GDL surface. For both models, results show that for higher velocities the droplet is more deformed.

Since the two forces acting on the droplet have opposite directions, the center of mass oscillates during droplet growth. The advancing and receding angles have oscillations as well, as displayed on Figure 6(b). In this simulation, as the droplet grows, the drag force deforms the droplet. As the drag increases, the difference between both angles also increases, which is represented as the hysteresis angle in $6(\mathrm{~b})$.

The two forces acting on the droplet are responsible for its oscillation. The bigger the droplet, the greater the drag force. The surface tension force is modelled using equation (3). The evolution of both forces over time is shown in Figure 6(c). One can observe that the drag force oscillates with small amplitude compared to the surface tension force. The main reason is that, for a certain droplet size, the frontal area does not change much with the oscillation of the center of mass $\mathrm{x}$-coordinate. The drag force has a quadratic growth due to its quadratic relationship with the droplet radius.

\subsection{Detachment time and force}

Using the proposed model, detachment times can be estimated based on the time it takes for a droplet to reach a critical hysteresis angle. Based on previous research, e.g. reference [18], it is estimated that a water droplet within airflow will detach from the surface when the contact angle hysteresis is greater than 30 degrees.

For a droplet in a fuel cell channel, the values in Table 2 are used to predict detachment while the maximum hysteresis angle is set to $30^{\circ}$. The proposed model then predicts detachment after $9.83 \mathrm{~s}$. When the droplet is subjected to airflow it oscillates and therefore is more prone to detachment. This result highlights the importance of a transient model for this problem. At high airflow rates, droplet geometry and droplet height will be independent of current density, and drops will not block the channel. However, at low air flow rates blockage is possible.

The detachment time is a function of the water injection rate [1], [7], [9], [8]. Several simulations have been done considering different values for the water inflow and two air velocities $\left(u_{1}=0.15 \mathrm{~ms}^{-1}, u_{2}=0.5 \mathrm{~ms}^{-1}\right)$ and the results are shown in Table 3. These air velocities correspond to the air flow rate for a stoichiometric ratio of one at two different current densities, $1 \mathrm{~A} \mathrm{~cm}^{-2}$ and $3 \mathrm{~A}$ $\mathrm{cm}^{-2}$, respectively.

The time values marked with an asterisk $\left({ }^{*}\right)$ correspond to those cases where the droplet blocks the channel before detachment. For the different water injection rates and the air flowing at $u_{2}=0.5 \mathrm{~ms}^{-1}$, the droplet detaches when it reaches a critical value of height, independently of liquid flow rate. 
Table 3: Detachment time, height, width, and area covered for different water injection rates $(\dot{Q})$ and air velocities $\left(u_{\text {air }}\right)$

\begin{tabular}{c|c|c|c|c|c|c|c|c}
\hline \multirow{2}{*}{$\dot{Q}\left[\mu \mathrm{l} \mathrm{s}^{-1}\right]$} & \multicolumn{2}{|c|}{$\mathrm{t}_{\mathrm{det}}[\mathrm{s}]$} & \multicolumn{2}{c|}{$\mathrm{A}_{\text {cov }}\left[\mathrm{mm}^{2}\right]$} & \multicolumn{2}{c|}{$\mathrm{h}[\mathrm{mm}]$} & \multicolumn{2}{c}{$\mathrm{w}[\mathrm{mm}]$} \\
\cline { 2 - 9 } & $u_{1}$ & $u_{2}$ & $u_{1}$ & $u_{2}$ & $u_{1}$ & $u_{2}$ & $u_{1}$ & $u_{2}$ \\
\hline \hline 0.01 & $49.32^{*}$ & 25.19 & \multirow{2}{*}{0.393} & & 0.853 & 0.683 & \multirow{2}{*}{1.000} & 0.8 \\
0.02 & $24.66^{*}$ & 12.58 & & 0.251 & & 0.681 & & 0.799 \\
0.04 & 11.52 & 6.31 & 0.376 & & 0.834 & 0.683 & 0.978 & 0.8 \\
0.08 & 5.31 & 4.60 & 0.356 & & 0.812 & 0.682 & 0.952 & 0.8 \\
\hline
\end{tabular}

\section{Conclusions}

A semi-analytical model of a water droplet emerging from a GDL pore in a cathode gas channel has been developed. Several numerical simulations have been done to obtain the value of the drag coefficient for different droplet sizes. For a droplet height to channel height ratio below $10 \%$ the drag coefficient has a linear relationship with the Reynolds number and hysteresis angle. For a droplet height to channel height ratio above $10 \%$ the drag force has a nonlinear relationship with the Reynolds number and the contact angle hysteresis.

From the analysis of the drag coefficient it can be concluded that using a spherical drop drag equation versus Reynolds number leads to underpredicted values of the drag coefficient and therefore the drag force. The results for the adhesion force show that the considered equation gives higher results than others obtained from a spring model [9], [16]. Previous approaches using a spring model might have underestimated the adhesion force by as much as $50 \%$. A lower value of the adhesion force means that the droplet oscillates with higher amplitude but with less frequency.

The value of the critical drag force corresponding to the droplet detachment is consistent with the experimental data in reference [18]. The detachment condition from reference [18] has been tested comparing the experimental data from reference [19]. Results show that the proposed model using a constant value of the critical hysteresis angle (Milne's condition) has good agreement with the experimental results.

The water injection rate study reveals that for low air velocities, the droplet blocks the channel before detaching the channel surface, whereas for high air velocities the droplet detaches when it reaches a critical height value. Applying this model to droplets in a fuel cell channel it has been found that at moderate flow rates and low airflow velocities droplets are likely to block the channels. Further, GDL coverage is at least $50 \%$ of the cross-sectional area, therefore it limits gas transport to the GDL.

\section{Acknowledgements}

This work has been supported by the FPI Research Grant BES-2011-047702 subject to the Spanish Project BIA2010-15880, from the Ministerio de Economía 


\section{Nomenclature}

$\Delta S \quad$ Surface variation of the droplet due to its deformation

$\Delta \quad$ Contact angle hysteresis

$\dot{Q} \quad$ Water volume inflow

$\gamma \quad$ Air-water surface tension coefficient

$\mu_{\text {air }} \quad$ Air viscosity

$\psi \quad$ Angle between the $x-y$ surface tension force and its $x$ component

$\rho_{\text {air }} \quad$ Air density

$\theta \quad$ Contact angle

$\theta_{\mathrm{A}} \quad$ Advancing contact angle

$\theta_{\mathrm{R}} \quad$ Receding contact angle

A Frontal projected area of the droplet

c Droplet chord length

$C_{\mathrm{D}} \quad$ Drag coefficient

$d x \quad$ Displacement of the droplet center of mass in the $x$ direction

$F_{\text {adh }} \quad$ Adhesion force acting on the droplet

$F_{\text {drag }}$ Adhesion force acting on the droplet

$F_{\mathrm{ST}} \quad$ Surface tension force

$H \quad$ Channel height

$h \quad$ Droplet height

$m \quad$ Mass of the droplet

$P \quad$ Droplet perimeter

$R \quad$ Droplet radius

$R e_{\mathrm{H}-\mathrm{h}}$ Reynolds number with the difference $H-h$ as the characteristic length

$R e_{\mathrm{h}} \quad$ Reynolds number with $h$ as the characteristic length

$u_{\text {air }} \quad$ Air velocity

$v_{\mathrm{CM}} \quad$ Velocity of the droplet center of mass

$x_{\mathrm{CM}} \quad x$-coordinate of the droplet center of mass

y Competitividad. 


\section{References}

[1] X. Zhu, P. Sui, N. Djilali, Three-dimensional numerical simulations of water droplet dynamics in a pemfc gas channel, Journal of Power Sources 181 (2008) 101-115.

[2] J. Carton, V. Lawlor, A. Olabi, C. Hochenauer, G. Zauner, Water droplet accumulation and motion in pem (proton exchange membrane) fuel cell mini-channels, Energy 39 (2012) 63-73.

[3] K. Chen, M. Hickner, D. Noble, Simplified models for predicting the onset of liquid water droplet instability at the gas diffusion layer/gas flow channel interface, International Journal of Energy Research 29 (12) (2005) 11131132.

[4] A. Theodorakakos, T. Ous, M. Gavaises, J. Nouri, N. Nikolopoulos, H. Yanagihara, Dynamics of water droplets detached from porous surfaces of relevance to pem fuel cells, Journal of Colloid and Interface Science 300 (2006) 673-687.

[5] T. Wu, N. Djilali, Experimental investigation of water droplet emergence in a model polymer electrolyte membrane fuel cell microchannel, Journal of Power Sources 208 (2012) 248-256.

[6] X. Zhu, Q. Liao, P. Sui, N. Djilali, Numerical investigation of water droplet dynamics in a low-temperature fuel cell microchannel: Effect of channel geometry, Journal of Power Sources 195 (2010) 801-812.

[7] X. Zhu, P. Sui, N. Djilali, Numerical simulation of emergence of a water droplet from a pore into a microchannel gas stream, Microfluid Nanofluid 4 (2008) 543-555.

[8] E. Kumbur, K. Sharp, M. Mench, Liquid droplet behavior and instability in a polymer electrolyte fuel cell flow channel, Journal of Power Sources 161 (2006) 333-345.

[9] A. Esposito, P. Polverino, C. Pianese, Y. Guezennec, A lumped model of single droplet deformation, oscillation and detachment on the gdl surface of a pem fuel cell, ASME 2010 8th International Fuel Cell Science, Engineering and Technology Conference.

[10] S. C. Cho, Y. Wanga, K. Chen, Droplet dynamics in a polymer electrolyte fuel cell gas flow channel: Forces, deformation, and detachment. I: Theoretical and numerical analyses, Journal of Power Sources 206 (2012) 119-128.

[11] C. Hirt, B. Nichols, Volume of Fluid (VOF) method for the dynamics of free boundaries, Journal of Computational Physics 39 (1981) 201-225.

[12] C. Extrand, Y. Kumagai, Liquid drops on an inclined plane: the relation between contact angles, drop shape, and retentive force, Journal of Colloid and Interface Science 170 (1995) 515-521. 
[13] Energy Systems Design Laboratory (ESDLab), University of Alberta, http://www. esdlab.mece. ualberta.ca/.

[14] P. Dadvand, R. Rossi, E. Oñate, An object-oriented environment for developing finite element codes for multi-disciplinary applications, Lab on a Chip 4 (2004) 257-264.

[15] J. Donea, A. Huerta, Finite Element Methods for Flow Problems, 1st Edition, John Wiley \& Sons, 2003.

[16] F. Celestini, R. Kofman, Vibration of submillimeter-size supported droplets, Physical Review 73 (1).

[17] C. Antonini, F. Carmona, E. Pierce, M. Marengo, A. Amirfazli, General methodology for evaluating the adhesion force of drops and bubbles on solid surfaces, Langmuir 25 (11) (2009) 6143-6154.

[18] A. Milne, A. Amirfazli, Drop shedding by shear flow or hydrophilic to superhydrophobic surfaces, Langmuir 25 (24) (2009) 14155-14164.

[19] F. Y. Zhang, X. G. Yang, C. Y. Wang, Liquid water removal from a polymer electrolyte fuel cell, Journal of The Electrochemical Society 153 (2) (2006) A225-A232. 